\title{
Shortcomings of short hairpin RNA-based transgenic RNA interference in mouse oocytes
}

\author{
Lenka Sarnova ${ }^{1,2}$, Radek Malik $^{1 *}$, Radislav Sedlacek$^{2}$, Petr Svoboda $^{1}$
}

\begin{abstract}
Background: RNA interference (RNAi) is a powerful approach to study a gene function. Transgenic RNAi is an adaptation of this approach where suppression of a specific gene is achieved by expression of an RNA hairpin from a transgene. In somatic cells, where a long double-stranded RNA (dsRNA) longer than 30 base-pairs can induce a sequence-independent interferon response, short hairpin RNA (shRNA) expression is used to induce RNAi. In contrast, transgenic RNAi in the oocyte routinely employs a long RNA hairpin. Transgenic RNAi based on long hairpin RNA, although robust and successful, is restricted to a few cell types, where long double-stranded RNA does not induce sequence-independent responses. Transgenic RNAi in mouse oocytes based on a shRNA offers several potential advantages, including simple cloning of the transgenic vector and an ability to use the same targeting construct in any cell type.
\end{abstract}

Results: Here we report our experience with shRNA-based transgenic RNAi in mouse oocytes. Despite optimal starting conditions for this experiment, we experienced several setbacks, which outweigh potential benefits of the shRNA system. First, obtaining an efficient shRNA is potentially a time-consuming and expensive task. Second, we observed that our transgene, which was based on a common commercial vector, was readily silenced in transgenic animals.

Conclusions: We conclude that, the long RNA hairpin-based RNAi is more reliable and cost-effective and we recommend it as a method-of-choice when a gene is studied selectively in the oocyte.

\section{Background}

RNA interference (RNAi) is a sequence-specific mRNA degradation induced by double stranded RNA (dsRNA). Briefly, long dsRNA is processed in the cytoplasm by RNase III Dicer into 20 - 22 bp long short interfering RNAs (siRNAs), which are loaded on the effector RNAinduced silencing complex (RISC). siRNAs serve as guides for cleavage of complementary RNAs, which are cleaved in the middle of the duplex formed between a siRNA and its cognate RNA (reviewed in detail in [1]).

RNAi is a widely used approach for inhibiting gene function in many eukaryotic model systems. Compared to other strategies for blocking gene functions, RNAi provides several advantages. It can be used to silence any gene, it is fast, relatively simple to use, and its cost is reasonably low. RNAi is usually induced either by

\footnotetext{
* Correspondence: malikr@img.cas.cz

'Department of Epigenetic Regulations, Institute of Molecular Genetics of the AS CR, Videnska 1083, CZ-14220 Prague 4, Czech Republic

Full list of author information is available at the end of the article
}

delivering siRNAs or long dsRNAs into cells or by expressing RNA-inducing molecules from a vector. A number of strategies was developed for tissue-specific and inducible RNAi, thus offering an attractive alternative to traditional gene targeting by homologous recombination.

RNAi became a favorable tool to block gene function also in mammalian oocytes. In fact, mouse oocytes were the first mammalian cell type where RNAi was used $[2,3]$. RNAi induced by microinjection of long dsRNA or siRNA into fully-grown germinal vesicle-intact (GV) oocytes is an excellent tool to study the role of dormant maternal mRNAs. These mRNAs are not translated before resumption of meiosis, so the stability of the protein product is not a factor influencing the efficiency of RNAi. In addition, resumption of meiosis can be delayed by compounds preventing reduction of cAMP levels in the GV oocyte, such as isobutylmethylxantine (IBMX) or milrinone, hence the period of mRNA degradation in microinjected oocytes can be prolonged for up to 48
C Biomed Central

C 2010 Sarnova et al; licensee BioMed Central Ltd. This is an Open Access article distributed under the terms of the Creative Commons Attribution License (http://creativecommons.org/licenses/by/2.0), which permits unrestricted use, distribution, and reproduction in any medium, provided the original work is properly cited. 


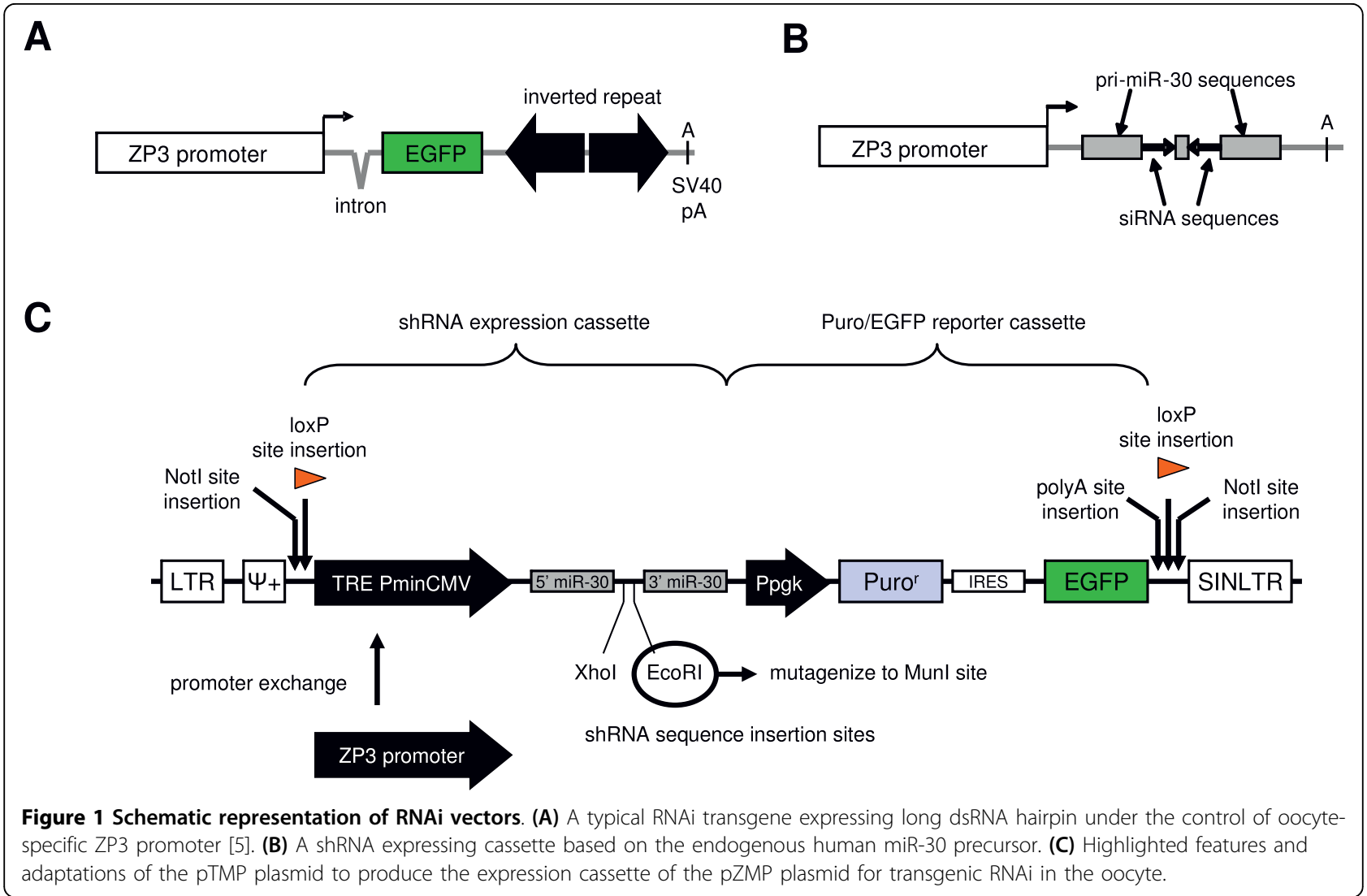

hours [4]. The ability to target also genes translated during oocyte growth has been greatly enhanced by development of transgenic RNAi based on oocyte-specific expression of long dsRNA hairpin (Figure 1A, [5]). In comparison to the traditional conditional knock-out, transgenic RNAi is simpler, cheaper, and can produce phenotypes of different severity, depending on the knockdown level $[5,6]$. At least ten genes were efficiently suppressed in the mouse oocyte using a long hairpinexpressing transgene ([7] and P.S., unpublished results). Transgenic RNAi based on long RNA hairpin expression, however, has two limitations. First, cloning an inverted repeat needed for long RNA hairpin expression may sometimes be a difficult task. Second, long dsRNA efficiently induces a specific RNAi effect only in a limited number of cell types (reviewed in [7]). Endogenous RNAi manifested by the presence of endogenous siRNAs derived from long dsRNA, was found only in oocytes and embryonic stem (ES) cells, an artificial cell type closely related to cells of the blastocyst stage [8-10]. Because dsRNA longer than $30 \mathrm{bp}$ has been reported to trigger the interferon response [11] and sequence-independent effects were observed in differentiated ES cells [12], induction of RNAi with expressed long hairpin RNA never acquired wider attention besides mouse oocytes.
We decided to develop and test a new transgenic RNAi vector for oocyte-specific short hairpin RNA (shRNA) expression, which would be compatible with RNAi vectors used in somatic cells and would be more versatile than the traditional transgenic RNAi design (Figure 1). First, a simple promoter swap would allow for using the same RNAi system for blocking genes in cultured cells or in tissues. Second, cloning shRNA-producing vector is easier when compared to cloning large inverted repeats. Third, a new vector would be compatible with different strategies to generate transgenic RNAi animals.

\section{Results}

\section{Vector design}

The RNAi targeting vector, named pZMP (Figure 1C) was based on pTMP and pLMP plasmids (Open Biosystems), which were selected as suitable starting vectors for producing a vector for transgenic RNAi in mouse oocyte. Vectors pTMP and pLMP allow for stable integration into the genome upon viral transduction and they carry suitable restriction sites for additional modifications. Furthermore, we needed a vector where shRNA expression would be driven by RNA polymerase II (pol II). The first shRNA systems were driven by pol III (reviewed in [13]). Pol II systems appeared later [14-17] 
since their development required better understanding of microRNA (miRNA) biology. miRNAs are genomeencoded small RNAs, which are loaded on the same effector complexes as siRNAs in mammalian cells [18].

Requirement for oocyte-specific expression dictated using a pol II-driven shRNA mimicking endogenous miRNA. The oocyte-specific expression of shRNA (Figure 1A) is controlled by the ZP3 promoter (hence pZMP), which is highly active during oocyte growth [19]. The transgenic cassette is flanked by LoxP sequences and NotI sites allowing for Cre-mediated insertion in the genome and simple release of the transgene from the plasmid for microinjection, respectively. Finally, the EcoRI site used for insertion of shRNA was mutated to MunI because there is another EcoRI site present in the ZP3 promoter. Since, MunI and EcoRI produce compatible overhangs the same oligonucleotides can be used for inserting shRNA into pTMP, pLMP and pZMP plasmids.

\section{Vector cloning and testing}

First, we compared pTMP and pLMP vectors with three other shRNA vectors, to assure that both parental vectors would offer robust silencing. pTMP and pLMP essentially differ in the promoter controlling shRNA expression. pLMP uses the constitutively active 5'LTR promoter, while the PTMP vector uses a modified CMV promoter allowing for tetracycline-inducible expression. Using a published shRNA sequence targeting firefly luciferase [20], we generated five different vectors targeting firefly luciferase sequence, and compared their efficiency in transiently transfected cell lines (Figure 2A). Our results showed that pTMP and pLMP vectors induce RNAi efficiently, when compared to other shRNA vectors.

Next, we modified pLMP and pTMP plasmids by inserting linkers with LoxP and NotI sites, which flank the expression cassette (Figure 1C). The functionality of LoxP sites was tested in E. coli strain expressing Cre recombinase (Figure $2 \mathrm{~B}$ ) and we also verified that LoxP insertion has no effect on the efficiency of RNAi induced by these vectors (Figure 2C). Subsequently, the ZP3 promoter from the published transgenic RNAi cassette [5] was inserted in the pLMP vector (Figure $1 \mathrm{C}$ ) and the NotI-flanked vector backbone was exchanged with the pTMP because it is modified to render the retrovirusintegrated 5' LTR transcriptionally inactive, in order to prevent interfering with the pol II promoter driving shRNA expression. The vector sequence was verified by sequencing. The functionality of PGK-driven puromycinIRES-EGFP reporter was tested in cell culture.

\section{Mos shRNA selection}

Mos dormant maternal mRNA was selected as the target for the new RNAi vector. Targeting Mos gene offers several advantages. First, Mos knock-out phenotype is manifested as sterility or subfertility, which is caused by parthenogenetic activation of eggs in otherwise normal animals $[21,22]$. This allows for simple scoring for the null phenotype and identification of potential non-specific effects of the PGK-driven reporter system in somatic cells. Second, maternal Mos has been targeted by microinjection of long dsRNA [2,3,23], siRNA [23] and by transgenic RNAi with long dsRNA [5,24], so there is a considerable volume of data for evaluating pZMP vector efficiency.

To silence Mos, we designed eight different shRNA sequences located within the Mos coding sequence (Figure 3A). Mos-targeting siRNAs were predicted by RNAi Codex database [25], BIOPREDsi [26], RNAxs [27] and RNAi Oligo Retriever [28] tools. Best scored siRNAs predicted by different algorithms were inserted in pLMP and pTMP vectors in the form of shRNA and were subsequently experimentally tested to find the most efficient constructs.

A Mos fragment, carrying homologous sequences to selected shRNAs, was inserted in the 3'UTR of Renilla luciferase and resulting reporter was used to estimate the inhibitory potential of individual shRNAs (Figure 3B). We also tested the strand selection of most efficient shRNAs to verify that the desired shRNA strand is efficiently loaded on the RISC. In this case, we used a Renilla luciferase reporter with the cognate Mos target sequence inserted in the antisense orientation. Our results suggested that the Mos mRNA targeting siRNA strand is specifically loaded on the RISC complex, while the other strand (so-called "passenger strand") had a negligible effect on the reporter (Figure 3C). This indicated an efficient loading of the correct siRNA strand. Based on these data, we have chosen the Mos_F shRNA sequence for further experiments and inserted it into the pZMP plasmid. Then, NotI-flanked transgenic cassette was released and, after purification, the linearized DNA fragment was used for transgenesis by pronuclear microinjection into once-cell embryos.

\section{Analysis of transgenic mice}

Upon embryo transfer, 56 founder $\left(F_{0}\right)$ mice were born. Six of these mice were positive for the transgene by PCR genotyping. One of the founder animals (\#840) never transmitted the transgene into the $F_{1}$ generation and one founder male (\#900) did not produce any progeny. $F_{0}$ mice from the remaining transgenic lines (\#819, \#835, \#892, and \#896) were fertile and transmitted the transgene. These lines were expanded and further examined. Interestingly, we noticed that the transgene transmission into the male progeny was reduced in all four lines (Table 1). Whether this unique sex-specific effect is caused by a particular transgene 


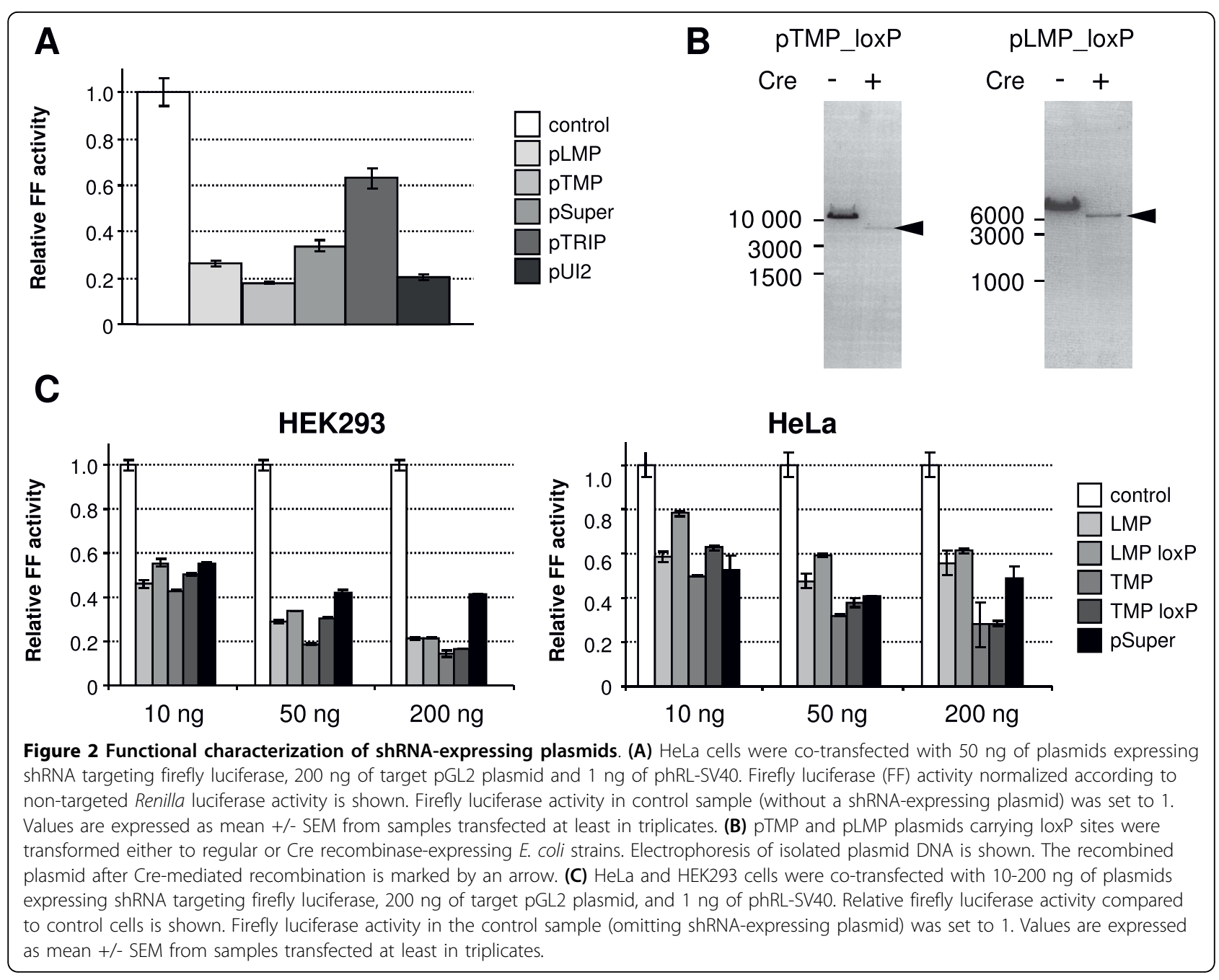

sequence, or is specific to disturbance of Mos expression $[29,30]$, or is an effect of a hemizygous locus in a homozygous genetic background is unknown and is currently under investigation.

Genotyping of transgenic mice should be facilitated by ubiquitous EGFP expression. However, none of the tails of $\mathrm{F}_{0}$ mice exhibited EGFP expression originating from the PGK-driven puromycin-IRES-EGFP reporter cassette in the transgene (Figure 4A). Likewise, none of the tested tissues in $F_{1}$ mice (brain, kidney, liver, spleen, testis, and oocytes) showed EGFP expression under the stereomicroscope (Figure 4A and 4B).

To test whether the reporter is completely silenced or the EGFP expression is below a detection limit of our microscope, we isolated tail fibroblasts from transgenic mice and their wild-type siblings and tested in culture their sensitivity to puromycin and assessed the transgene expression by RT-PCR and EGFP fluorescence by flow cytometry and fluorescent microscopy. Results of these experiments confirmed that the reporter cassette in the transgene is silenced in fibroblasts of $F_{1}$ mice of all available transgenic lines (Figure $4 \mathrm{C}$ ). We also tried to change the genetic background by crossing the $\mathrm{C} 57 \mathrm{Bl} / 6$ transgenic animals with BALB-C mice but it did not help to reactivate the silenced reporter in somatic cells (data not shown). This effect is likely due to the epigenetic silencing of the transgene because PCR analysis of genomic DNA showed that the transgene is intact. In addition, transfection of the purified transgene into 3T3 fibroblasts resulted in EGFP expression (Figure 4C) and puromycin resistance (data not shown), further supporting the idea that the transgene is epigenetically silenced.

Although silencing of the reporter cassette in the transgene was disappointing, we analyzed fertility, frequency of parthenogenetic activation, and Mos mRNA levels in four available transgenic lines because the shRNA was driven by a different promoter than the puromycin-EGFP reporter and the germline undergoes 


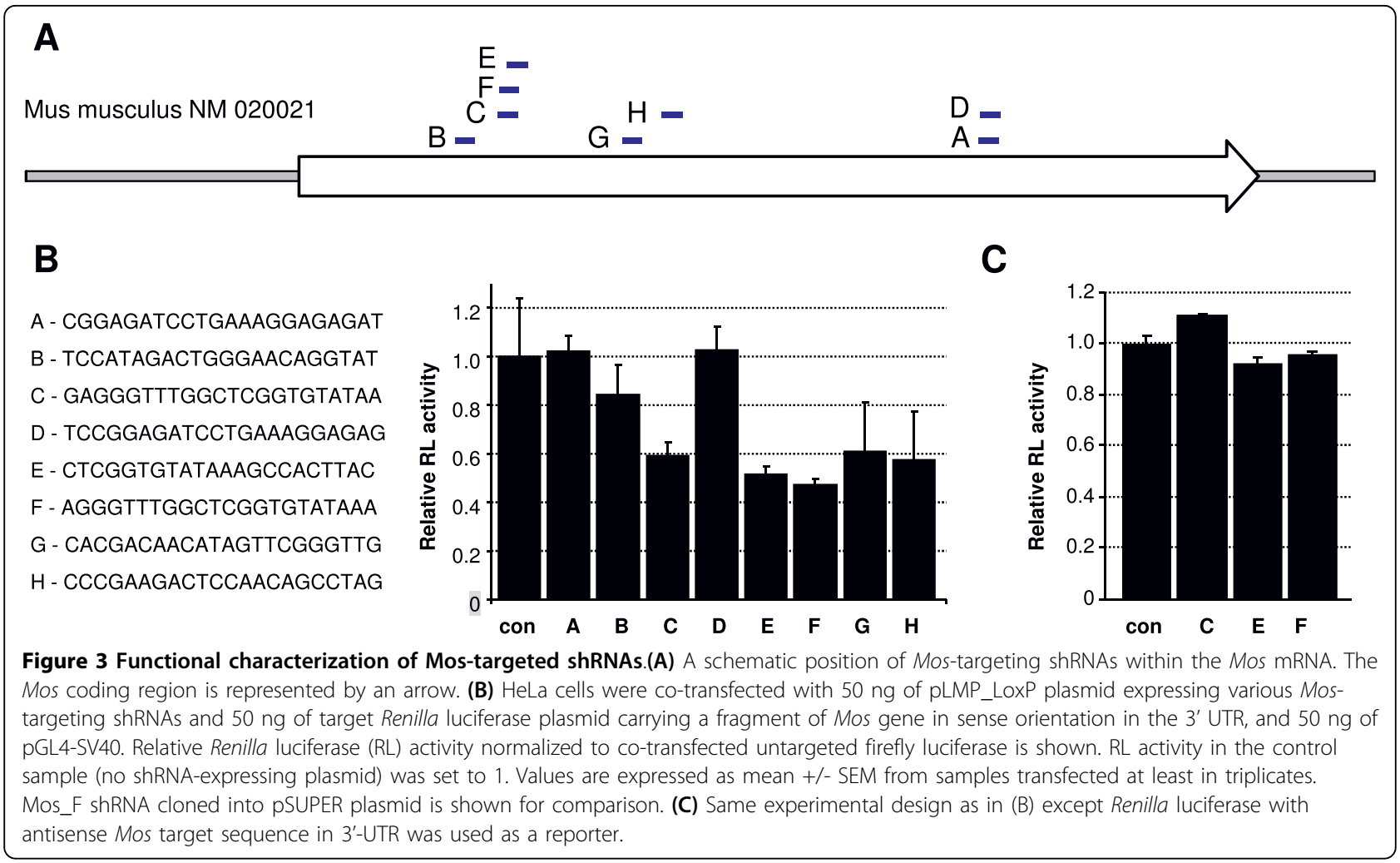

cycles of epigenetic reprogramming, providing a chance that the transgene would be active in the oocyte. However, oocytes of transgenic animals did not exhibit parthenogenetic activation. Single-cell quantitative realtime PCR (qPCR) showed a possible down-regulation of Mos mRNA (up to 2-fold) in transgenic lines \#819, \#835, and \#892 compared to wild-type controls (Figure $5 \mathrm{~A})$, but it was not statistically significant when considering the variability of mRNA level in individual oocytes. However, it is possible that a mild down-regulation was induced in the line \#835 where we observed the lowest Mos mRNA level and qPCR analysis suggested a low level of shRNA expression (Figure 5B). These data indicate that epigenetic silencing affects the whole transgene, leading to low shRNA expression, which in turn is unable to target Mos mRNA efficiently.

Table 1 Overview of $F_{1}$ and $F_{2}$ progeny of transgenic founder animals

\begin{tabular}{|c|c|c|c|c|c|c|c|}
\hline \multirow[t]{2}{*}{ Sex } & \multirow[t]{2}{*}{ Transgene } & \multicolumn{4}{|c|}{ Number of pups in individual lines } & \multirow[t]{2}{*}{ Sum } & \multirow[t]{2}{*}{$\%$} \\
\hline & & \#819 & \#835 & \#892 & \#896 & & \\
\hline \multirow[t]{2}{*}{$M$} & + & 8 & 5 & 8 & 13 & 34 & $33.3 \%$ \\
\hline & - & 19 & 12 & 16 & 21 & 68 & $66.6 \%$ \\
\hline \multirow[t]{2}{*}{$F$} & + & 20 & 4 & 20 & 15 & 59 & $54.6 \%$ \\
\hline & - & 10 & 6 & 15 & 18 & 49 & $45.4 \%$ \\
\hline
\end{tabular}

\section{Discussion}

Long hairpin RNA expression has been a preferred solution for specific gene inhibition by RNAi during oocyte growth and oocyte-to-zygote transition. At least ten different genes were targeted by this approach and strong mRNA knockdown was observed in all cases ([7] and P. S., unpublished results). Successful knockdown in the oocytes with transgenic short hairpin systems was reported in the mouse using Cre-recombination-activated pol III promoter-driven shRNA [31]. A ZP3 promoter-driven shRNA expression in Steppe Lemming oocytes induced an efficient RNAi [32], suggesting that miRNA-like shRNA biogenesis is intact in rodent oocytes.

Here, we show that experiments with pol II-driven miRNA-like shRNA system did not meet expectations and raised questions whether such a system represents a more versatile and economical alternative to the long hairpin RNA-based approach. The expected benefit of the shRNA system, a simple production of the targeting vector, turned out to be correct and targeting vectors were easily produced in a single cloning step. Easy production of different targeting vectors facilitates testing different siRNA sequences in transient cell culture transfections before producing transgenic animals. This used to be an advantage over the long hairpin RNA system, where targeting efficiency of transgenic constructs 


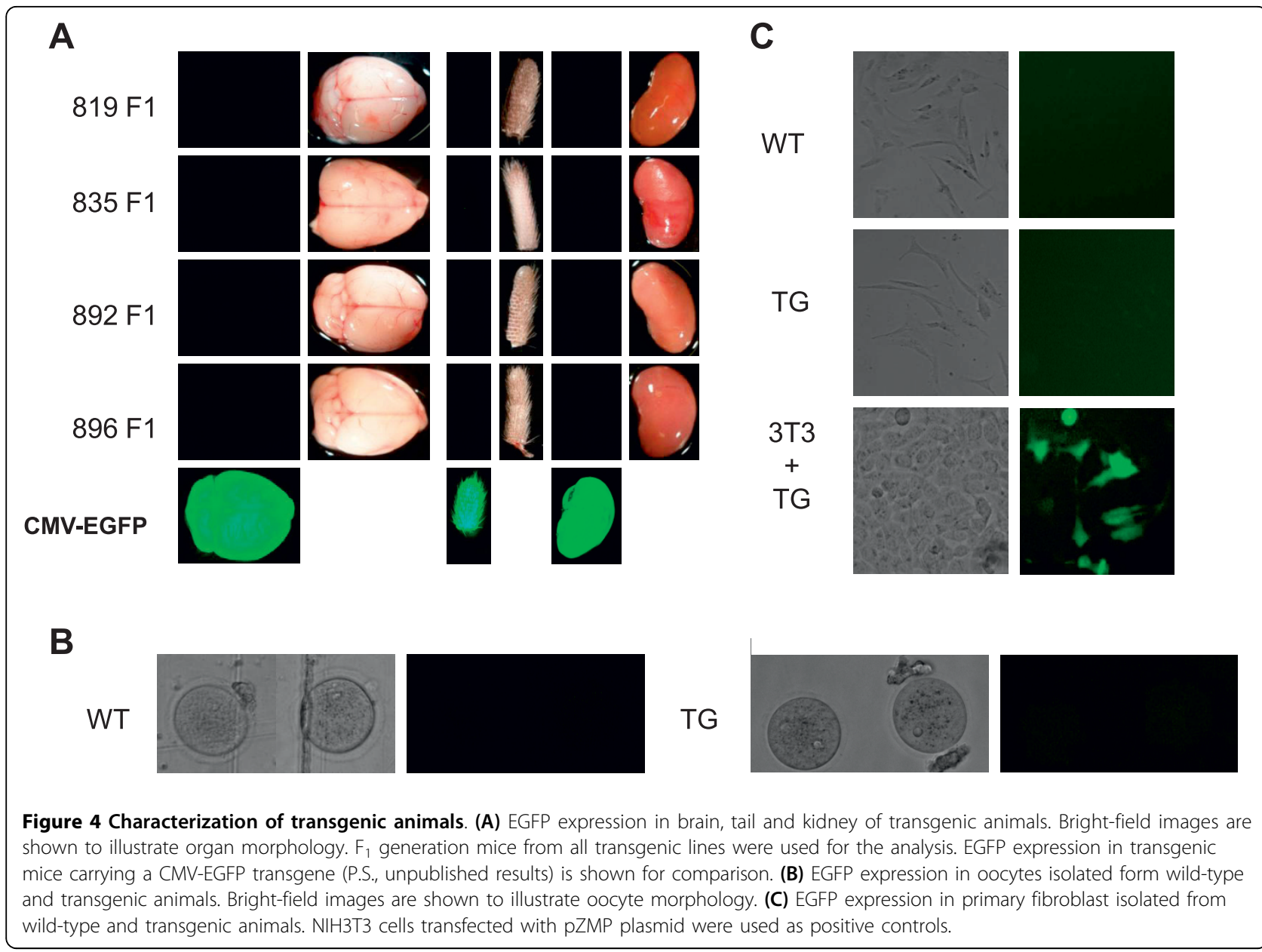

could be tested only by microinjecting them into incompetent oocytes. To circumvent this problem, different strategies are available now that simplify cloning of long inverted repeats [33] and, in our experience, the transgenic approach with long hairpin RNA is reliable enough that, upon verifying the correct structure of the transgene by sequencing, we routinely proceed directly to production of transgenic animals.

Thus, designing and cloning functional shRNAs is not a significant advantage over producing the traditional long hairpin-expressing transgene. A shRNA with a defined sequence exhibits sequence-specific off-target effects. Thus, one needs at least two different shRNAs and/or other means to assure that off-targeting will not interfere with interpretation of data $[34,35]$. This complicates the production of transgenic lines because, ideally, one would need to have different transgenic lines expressing different shRNAs targeting the same gene. In addition, obtaining effective shRNAs may also represent a problem. While testing eight different shRNAs designed by the best available algorithms [25-28], we found just two good shRNAs with $\sim 50 \%$ knockdown effects in a transient reporter assay. This issue will be reduced in the future as more verified shRNA sequences will become available. Still, obtaining verified shRNAs against oocyte-specific genes might represent a problem.

Transgenic RNAi with shRNA is not more economical. Testing different shRNAs (eight in our case) required custom synthesis of eight long oligonucleotides and cloning of eight targeting vectors plus cloning one targeted reporter vector because the targeted gene was oocyte-specific, hence not expressed in common cell lines. This actually made the total cost of the experiment higher when compared to long hairpin transgenes.

In any case, theoretical advantages became irrelevant during the disappointing pilot experiment, where all transgenic lines produced by traditional pronuclear microinjection carried completely silenced transgenes in all tissues in the $F_{0}$ generation already. In contrast, long hairpin RNAi transgenes produced by pronuclear microinjection in the same transgenic facility, in the same genetic background, and carrying the same ZP3 promoter induced strong knock-down effects in oocytes (PS, unpublished results). 


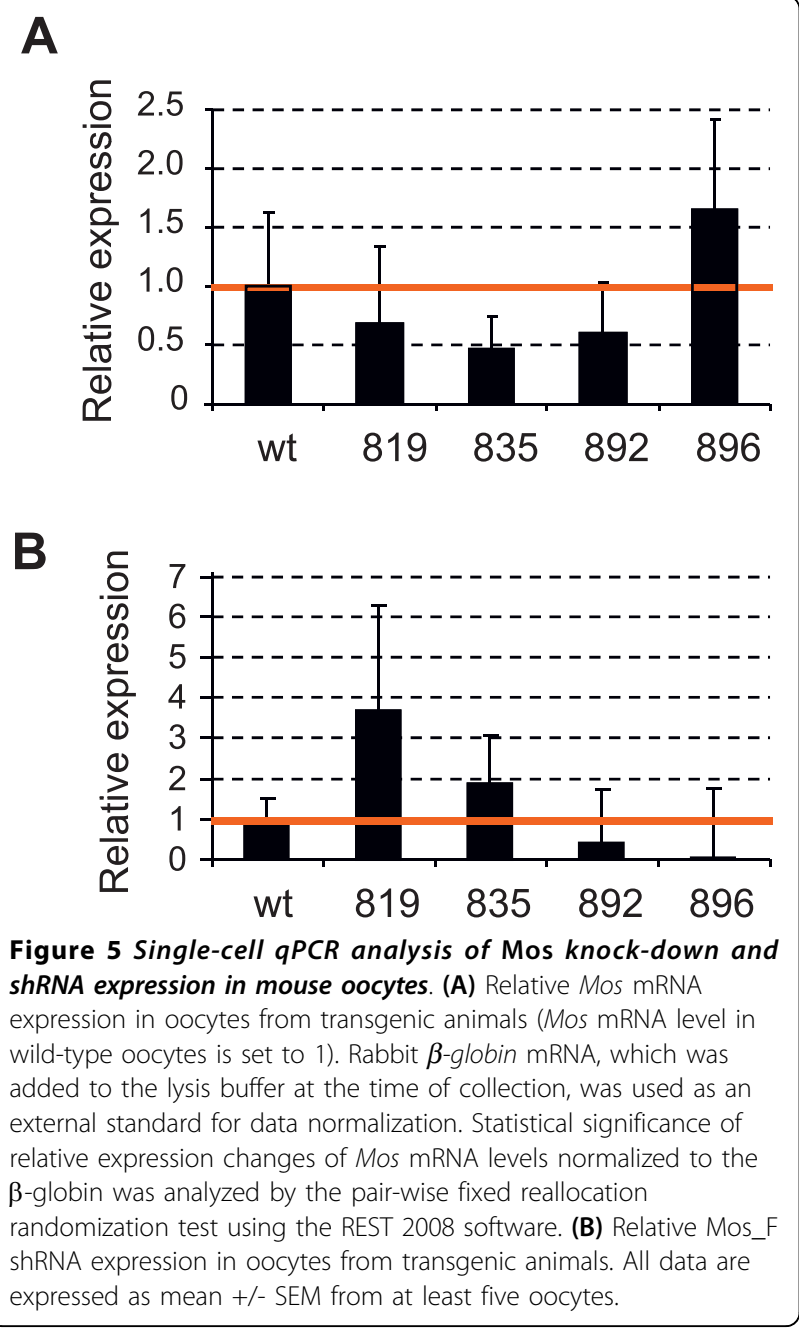

Available evidence points towards the reason for silencing being associated with the shRNA transgene sequence/structure. First, we have never seen such a rapid and complete silencing of a transgene in all tissues of $F_{0}$ animals and their progeny with other transgenes. This silencing is really striking considering the same transgene produces puromycin resistance and EGFP expression when transiently transfected in mouse NIH3T3 cells (Figure 4C). We speculate that, while it is tolerated in cells during transient transfection, the unusual structure of the transgene (flanking with short inverted repeats of LoxP sites and the absence of introns) and expression of unspliced bicistronic reporter mRNAs carrying a viral IRES contribute to its silencing when the transgene is integrated in the genome of an animal. Thus, our data show that optimization of shRNA-expressing transgenes design is needed and that intron-less transgenic cassette compatible with retroviral transgenesis might be suboptimal for transgenic RNAi in the mouse.

\section{Conclusions}

The oocyte-specific transgenic RNAi mediated by shRNA does not have any significant advantage in terms of labour, price, knockdown efficiency, and specificity. Transgenic RNAi with shRNA in the oocyte might represent an advantage only in the case when the same gene is being studied in the oocyte and somatic cells. In other cases, transgenic RNAi with long hairpin RNA appears to be a better approach. Current strategies for cloning long inverted repeats make the production of long hairpin-expressing transgenes feasible and costeffective $[33,36]$. To our knowledge, all transgenic RNAi experiments with long hairpin-expressing transgenes yielded transgenic lines with strong silencing including phenocopying the knockout phenotypes. Moreover, detailed analysis of non-specific effects revealed remarkable specificity of transgenic RNAi induced by long hairpin RNA [24], presumably because off-target effects are minimized by processing long dsRNA into a pool of siRNAs with different sequences [37].

\section{Methods}

Plasmids

\section{Renilla-Mos reporters}

Renilla-Mos reporters were generated from phRL_SV40 (Promega) by inserting a Mos fragment into the Renilla 3'-UTR. The Mos fragment was amplified by PCR from genomic DNA using Mos_XbaI_Fwd and Mos_XbaI_Rev primers (see additional file 1: A list of oligonucleotide sequences used in this study). PCR product was cleaved and inserted into the XbaI site in phRL_SV40 to produce phRL_SV40_mMos and phRL_SV40_ asMos reporters where the Mos fragment was inserted in a sense and an antisense orientation, respectively.

\section{pLMP and pTMP shRNA plasmids}

For each shRNA to be inserted into pLMP and pTMP plasmid, one long oligonucleotide was synthesized (Sigma-Aldrich). Each oligonucleotide was used as a template for PCR (performed according to the manufacturer's instructions) using LMP_oligo.fwd and LMP_oligo.rev primers. Resulting PCR product was digested by EcoRI and XhoI and cloned into the target plasmid digested by XhoI and EcoRI. All plasmids were verified by sequencing.

\section{pZMP and pZMP-Mos_F}

The pZMP vector was derived from pTMP and pLMP plasmids as follows. 5' and 3' LoxP and NotI sites flanking the transgenic cassette were sequentially inserted into BglII and SalI sites, respectively, in pLMP and pTMP in a form of in vitro synthesized annealed linkers (5'loxP.fwd/rev and 3'loxP.fwd/rev, respectively) producing pLMP_LoxP and pTMP_LoxP. Subsequently, the EcoRI site for shRNA cloning in the pLMP_LoxP vector was mutagenized to the MunI site using Quick Change 
II XL Site-Direct Mutagenesis Kit (Stratagene) according to manufacturer's instructions using LMP_MunI.fwd and LMP_MunI.rev primers.

The ZP3 promoter was amplified from the original transgenic RNAi vector [5] by PCR using primers ZP3_BglII_Fwd and ZP3_BglII_Rev using a Pfu DNA polymerase. The ZP3 promoter-carrying PCR fragment was cleaved by BglII and inserted in the BglII site in the pLMP_LoxP plasmid to get pLMP_LoxP_ZP3 plasmid. The correct orientation of the ZP3 promoter and the absence of mutations were verified by sequencing. Finally, the NotI-flanked CMV-TRE-Puromycin-EGFP cassette in the PTMP_LoxP plasmid was replaced by the NotI site-flanked ZP3 cassette from pLMP_LoxP_ZP3 plasmid to produce the pZMP vector ready for shRNA insertion. The reason for this strategy was that the pTMP_LoxP plasmid did not contain suitable restriction sites for direct insertion of the ZP3 promoter while the pLMP is not a self-inactivating (SIN) retroviral vector and strong promoter present in the 5'LTR region of pLMP would have undesirable effects on shRNA expression.

Finally, Mos_F shRNA was inserted in the pZMP to produce pZMP-Mos_F plasmid. The transgenic cassette $(\sim 4.5 \mathrm{~kb})$ was released by NotI digest, isolated by Gel Extraction Kit (Qiagen), and purified twice using DNA Clean \& Concentrator kit (Zymo Research). The cassette purity and integrity was verified by agarose gel electrophoresis before it was submitted to the transgenic facility.

\section{Other plasmids}

Cloning of shRNAs targeting firefly luciferase (pGL2, Promega) into pLMP, pTMP and pTRIPZ plasmids (Open Biosystems) was performed as described above using $\mathrm{FL} \_1$ primer as a template. The insert for cloning into pSuper vector (OligoEngine) was prepared by annealing oligonucleotides FL_2 and FL_3 and cloning them into BglII and HindIII sites of target vector according to the manufacturer's instructions. A hairpin cloned into the UI2-GFP-SIBR vector [14] was prepared by annealing oligonucleotides FL_4 and FL_5 (see additional file 1: A list of oligonucleotide sequences used in this study). Annealed oligonucleotides were cloned into BpiI-cleaved vector. All plasmids were verified by sequencing.

\section{Cell culture \\ Transformed cell lines}

HeLa, HEK293, and NIH3T3 cells were cultured in Dulbecco's Modified Eagle medium (DMEM, Sigma) supplemented with $10 \%$ fetal bovine serum (FBS, Gibco), Penicillin $100 \mathrm{U} / \mathrm{ml}$ and Streptomycin $100 \mu \mathrm{g} / \mathrm{ml}$ (Gibco). For transfection, cells were seeded in 24-well plates at the initial density 30,000 (HeLa and NIH3T3) or 60,000 (HEK293) cells per well in $0.5 \mathrm{ml}$ of culture medium. 24 hours later, cells were transfected with 500 ng of plasmid DNA per well. TurboFect (Fermentas) was used as the transfection reagent. pBluescript (Stratagene) was used to equalize the total amount of DNA per transfection. A $1 \mathrm{ml}$ aliquot of fresh culture media was added 6 hours post-transfection. Each transfection was performed at least in duplicates. Cells were collected 48 hours post-transfection and used for analysis.

\section{Primary tail fibroblasts culture and puromycin selection}

Primary fibroblasts were prepared from tail biopsies by collagenase treatment as described previously [38]. Primary tail fibroblasts from transgenic and wild type mice were cultured in DMEM supplemented with $10 \%$ FBS and Penicillin/Streptomycin at $37^{\circ} \mathrm{C}$ and $5 \% \mathrm{CO}_{2}$ for at least five days. Before experiment, medium was changed and puromycin was added to the final concentration of $2.5 \mu \mathrm{g} / \mathrm{ml}$. Cell culture was continued for additional 2 days until the control cells from wild-type mice died.

\section{Dual Luciferase assay}

For luciferase assays, cells were typically transfected with 50-250 ng of a firefly luciferase coding plasmid (pGL4SV40 or pGL2), $1 \mathrm{ng}$ of a Renilla luciferase reporter plasmid, $50 \mathrm{ng}$ of a tested hairpin-coding vector, and pBluescript up to the total DNA amount $500 \mathrm{ng}$ per well. In some experiments, different concentrations of a tested plasmid (20 - $450 \mathrm{ng}$ ) were used. Control transfection did not include the shRNA-expressing vector. Cells were harvested 48 hours post-transfection and lysed with $150 \mu \mathrm{l}$ of Passive Lysis Buffer (Promega). Protein amount in lysates was quantified by Protein Assay Dye Reagent (Bio-Rad) according to the manufacturer's protocol. A $10 \mu \mathrm{l}$ aliquot of each lysate was pipetted into a 96-well plate and luciferase activity was measured using a Dual-Luciferase Reporter Assay System (Promega) according to the manufacturer's instructions. The measurement was performed on the Modulus Microplate luminometer (Turner BioSystems).

\section{Mice}

\section{Production of transgenic founders}

All animal experiments were approved by the Institutional Animal Use and Care Committees and were in agreement with Czech law and NIH (National Institutes of Health) guidelines. Transgenic mice were produced in the Transgenic core facility of the Institute of Molecular Genetics Academy of Science of the Czech Republic. Briefly, fertilized donor oocytes were obtained from super-ovulated 3-4 weeks old $\mathrm{C} 57 \mathrm{Bl} / 6 \mathrm{~N}$ females (Charles Rivers Laboratories). Hormonal stimulation was carried out as follows: 5U of Pregnant Mare's Serum Gonadotropine (PMSG/Folligon; Intervet) was injected into peritoneum. Forty-five hours later, $5 \mathrm{U}$ of human 
Choriogonadotropine (HCG, Sigma) was injected into peritoneum and mice were mated with $\mathrm{C} 57 \mathrm{Bl} / 6 \mathrm{~N}$ males. One day later, one-cell stage embryos were isolated from plugged females. Pronuclear injection (PNI) of transgene DNA into male pronucleus was performed. Embryo transfer was performed either at one-cell stage directly after PNI or at the two-cell stage after an overnight culture depending on the amount of foster mice available on a specific day. Pseudopregnant CD1 females were used as foster mothers. Females were paired with vasectomized CD1 males (for optimal stimulation of the female) a night before the transfer. Embryos were transferred into the oviduct (15-25 embryos per recipient, into one or both oviducts) under sterile conditions in SPF (specified pathogen free) area of animal house. CD1 mice were obtained from an in-house breeding.

\section{Genotyping}

The tail biopsies were obtained from 3-4 weeks old mice. GFP expression was analyzed by fluorescent stereomicroscope SZX16 (Olympus). Genotyping was performed by PCR and resulting products were analyzed by electrophoresis on $1.5 \%$ agarose gels.

\section{Oocyte isolation and culture}

Fully-grown GV-intact oocytes were obtained from eight-week old mice 44 hours after superovulation by intraperitoneal injection of $0.1 \mathrm{ml}$ (5 units) of PMSG (Folligon; Intervet). Oocytes were collected into M2 medium supplemented with $4 \mu \mathrm{g}$ of isobutylmethylxanthine (IBMX, $200 \mathrm{mM}$ ) to prevent resumption of meiosis. Cumulus cells were removed with a thin glass capillary. Isolated oocytes were either immediately analyzed by microscopy or washed twice in PBS and lysed for single-cell qPCR analysis. GV oocytes used for meiotic maturation were washed five-times in M2 medium without IBMX and cultured overnight in CZB medium supplemented with glutamine ( $5 \mu \mathrm{l}$ of $3 \%$ glutamine per $1 \mathrm{ml} \mathrm{CZB)[39].}$

\section{Quantitative real-time RT-PCR (qPCR)}

mRNA expression in oocytes was analyzed by single-cell qPCR [40]. Briefly, individual oocytes were washed in PBS and placed separately in $5 \mu \mathrm{l}$ of water. $1 \mu \mathrm{g}$ of stuffer rRNA (16S + 23S, Roche) and 15 pg of external standard rabbit $\beta$-globin mRNA (Sigma) were added to each sample. All samples were snap-frozen and stored at $-80^{\circ}$ $\mathrm{C}$ until further processing. Before qPCR, samples were incubated at $85^{\circ} \mathrm{C}$ for 5 minutes to lyse oocytes and then were placed on ice. $1 \mu \mathrm{l}$ of Oligo(dT) primer (50 $\mu \mathrm{M})$ or random hexanucleotides (Fermentas) and water up to $13 \mu \mathrm{l}$ were added to all samples. mRNA was reverse transcribed using RevertAid M-MuLV Reverse transcriptase (Fermentas). Reverse transcriptase was omitted in control (-RT) samples. Resulting cDNA was diluted 3:2 with water and a $3 \mu \mathrm{l}$ aliquot was used as a template for qPCR. qPCR was performed on the iQ5 machine (Bio-Rad) using Maxima SYBR Green qPCR Master Mix (Fermentas). Specific primers for mouse Mos and rabbit $\beta$-globin mRNAs were used (see additional file 1: A list of oligonucleotide sequences used in this study). qPCR data were analyzed by the iQ5 software (Bio-Rad) and values of crossing points (CPs) were evaluated for each reaction. PCR efficiency was calculated for each individual reaction using the exponential regression model [41] and CPs values were corrected accordingly. Statistical significance of relative expression changes of Mos mRNA levels normalized to the external $\beta$-globin standard was analyzed by the pair-wise fixed reallocation randomization test using the REST 2008 software [42].

\section{Additional material}

Additional file 1: A list of oligonucleotide sequences used in this study. A table listing sequences of all oligonucleotides used in this study.

\section{Acknowledgements}

We thank David L. Turner for the pUI2 vector and the staff of the Transgenic core facility of the Institute of Molecular Genetic AS CR and the animal facility for assistance with transgenic mice. This research was supported by the EMBO SDIG program, ME09039 grant, and the Purkynje Fellowship to PS.

\section{Author details}

${ }^{1}$ Department of Epigenetic Regulations, Institute of Molecular Genetics of the AS CR, Videnska 1083, CZ-14220 Prague 4, Czech Republic. ²Department of Transgenic Models of Diseases, Institute of Molecular Genetics of the AS CR, Videnska 1083, CZ-14220 Prague 4, Czech Republic.

\section{Authors' contributions}

LS performed all the experiments. RM participated in the design of the study and data analysis. RS participated in the production of transgenic animals. PS designed and coordinated the study. RM and PS wrote the manuscript. All authors read and approved the final manuscript.

\section{Competing interests}

The authors declare that they have no competing interests.

Received: 22 July 2010 Accepted: 12 October 2010

Published: 12 October 2010

\section{References}

1. Kim VN, Han J, Siomi MC: Biogenesis of small RNAs in animals. Nat Rev Mol Cell Biol 2009, 10(2):126-139.

2. Svoboda $P$, Stein $P$, Hayashi $H$, Schultz RM: Selective reduction of dormant maternal mRNAs in mouse oocytes by RNA interference. Development 2000, 127(19):4147-4156.

3. Wianny F, Zernicka-Goetz M: Specific interference with gene function by double-stranded RNA in early mouse development. Nat Cell Biol 2000, 2(2):70-75.

4. Stein P: Microinjection of dsRNA into fully-grown mouse oocytes. Cold Spring Harb Protoc 2009, 2009(1):pdb prot5132.

5. Stein P, Svoboda P, Schultz RM: Transgenic RNAi in mouse oocytes: a simple and fast approach to study gene function. Dev Biol 2003, 256(1):187-193. 
6. Fedoriw AM, Stein P, Svoboda P, Schultz RM, Bartolomei MS: Transgenic RNAi reveals essential function for CTCF in $\mathrm{H} 19$ gene imprinting. Science 2004, 303(5655):238-240.

7. Svoboda P: RNA silencing in mammalian oocytes and early embryos. Curr Top Microbiol Immunol 2008, 320:225-256.

8. Babiarz JE, Ruby JG, Wang Y, Bartel DP, Blelloch R: Mouse ES cells express endogenous shRNAs, siRNAs, and other Microprocessor-independent, Dicer-dependent small RNAs. Genes Dev 2008, 22(20):2773-2785.

9. Tam OH, Aravin AA, Stein P, Girard A, Murchison EP, Cheloufi S, Hodges E, Anger M, Sachidanandam R, Schultz RM, et al: Pseudogene-derived small interfering RNAs regulate gene expression in mouse oocytes. Nature 2008, 453(7194):534-538

10. Watanabe T, Totoki $Y$, Toyoda A, Kaneda M, Kuramochi-Miyagawa S, Obata Y, Chiba H, Kohara Y, Kono T, Nakano T, et al: Endogenous siRNAs from naturally formed dsRNAs regulate transcripts in mouse oocytes. Nature 2008, 453(7194):539-543.

11. Wang Q, Carmichael GG: Effects of length and location on the cellular response to double-stranded RNA. Microbiol Mol Biol Rev 2004, 68(3):432-452.

12. Yang S, Tutton S, Pierce E, Yoon K: Specific double-stranded RNA interference in undifferentiated mouse embryonic stem cells. $\mathrm{Mol} \mathrm{Cell}$ Biol 2001, 21(22):7807-7816.

13. McManus MT, Petersen CP, Haines BB, Chen J, Sharp PA: Gene silencing using micro-RNA designed hairpins. Rna 2002, 8(6):842-850.

14. Chung KH, Hart CC, Al-Bassam S, Avery A, Taylor J, Patel PD, Vojtek AB, Turner DL: Polycistronic RNA polymerase II expression vectors for RNA interference based on BIC/miR-155. Nucleic Acids Res 2006, 34(7):e53.

15. Dickins RA, McJunkin K, Hernando E, Premsrirut PK, Krizhanovsky V, Burgess DJ, Kim SY, Cordon-Cardo C, Zender L, Hannon GJ, et al: Tissuespecific and reversible RNA interference in transgenic mice. Nat Genet 2007, 39(7):914-921.

16. Xia XG, Zhou H, Samper E, Melov S, Xu Z: Pol II-expressed shRNA knocks down Sod2 gene expression and causes phenotypes of the gene knockout in mice. PLoS Genet 2006, 2(1):e10.

17. Zeng $Y$, Wagner EJ, Cullen BR: Both natural and designed micro RNAs can inhibit the expression of cognate mRNAs when expressed in human cells. Mol Cell 2002, 9(6):1327-1333.

18. Meister $G$, Landthaler $M$, Dorsett $Y$, Tuschl T: Sequence-specific inhibition of microRNA- and siRNA-induced RNA silencing. Rna 2004, 10(3):544-550.

19. Millar SE, Lader E, Liang LF, Dean J: Oocyte-specific factors bind a conserved upstream sequence required for mouse zona pellucida promoter activity. Mol Cell Biol 1991, 11(12):6197-6204.

20. Paddison PJ, Caudy AA, Bernstein E, Hannon GJ, Conklin DS: Short hairpin RNAs (shRNAs) induce sequence-specific silencing in mammalian cells. Genes Dev 2002, 16(8):948-958.

21. Colledge WH, Carlton MB, Udy GB, Evans MJ: Disruption of c-mos causes parthenogenetic development of unfertilized mouse eggs. Nature 1994, 370(6484):65-68

22. Hashimoto N, Watanabe N, Furuta $Y$, Tamemoto H, Sagata N, Yokoyama M, Okazaki K, Nagayoshi M, Takeda N, Ikawa Y, et al: Parthenogenetic activation of oocytes in c-mos-deficient mice. Nature 1994, 370(6484):68-71.

23. Kim MH, Yuan $X$, Okumura S, Ishikawa F: Successful inactivation of endogenous Oct-3/4 and c-mos genes in mouse preimplantation embryos and oocytes using short interfering RNAs. Biochem Biophys Res Commun 2002, 296(5):1372-1377.

24. Stein $P$, Zeng F, Pan $H$, Schultz RM: Absence of non-specific effects of RNA interference triggered by long double-stranded RNA in mouse oocytes. Dev Biol 2005, 286(2):464-471.

25. Olson A, Sheth N, Lee JS, Hannon G, Sachidanandam R: RNAi Codex: a portal/database for short-hairpin RNA (shRNA) gene-silencing constructs. Nucleic Acids Res 2006, , 34 Database: D153-157.

26. Huesken D, Lange J, Mickanin C, Weiler J, Asselbergs F, Warner J, Meloon B, Engel S, Rosenberg A, Cohen D, et al: Design of a genome-wide siRNA library using an artificial neural network. Nat Biotechnol 2005, 23(8):995-1001.

27. Tafer H, Ameres SL, Obernosterer G, Gebeshuber CA, Schroeder R, Martinez J, Hofacker IL: The impact of target site accessibility on the design of effective siRNAs. Nat Biotechnol 2008, 26(5):578-583.
28. Paddison PJ, Cleary M, Silva JM, Chang K, Sheth N, Sachidanandam R, Hannon GJ: Cloning of short hairpin RNAs for gene knockdown in mammalian cells. Nat Methods 2004, 1(2):163-167.

29. Cao SF, Li D, Yuan Q, Guan X, Xu C: Spatial and temporal expression of cmos in mouse testis during postnatal development. Asian J Androl 2008, 10(2):277-285.

30. Higgy NA, Zackson SL, van der Hoorn FA: Cell interactions in testis development: overexpression of c-mos in spermatocytes leads to increased germ cell proliferation. Dev Genet 1995, 16(2):190-200.

31. Coumoul X, Li W, Wang RH, Deng C: Inducible suppression of Fgfr2 and Survivin in ES cells using a combination of the RNA interference (RNAi) and the Cre-LoxP system. Nucleic Acids Res 2004, 32(10):e85.

32. Zhuang SZ, Li XJ, Zhang AL, Ma J, Zheng YH, Zhang FC: Transgenic RNAimediated reduction of LZP3 in Lagurus lagurus oocytes results in decreased fertilization ability in IVF. Mol Biol Rep 2010, 37(3):1253-1259.

33. Svoboda P: Cloning a transgene for transgenic RNAi in mouse oocytes. Cold Spring Harb Protoc. 2009, 2009(1):pdb prot5134.

34. Echeverri CJ, Beachy PA, Baum B, Boutros M, Buchholz F, Chanda SK, Downward J, Ellenberg J, Fraser AG, Hacohen N, et al: Minimizing the risk of reporting false positives in large-scale RNAi screens. Nat Methods 2006, 3(10):777-779.

35. Svoboda P: Off-targeting and other non-specific effects of RNAi experiments in mammalian cells. Curr Opin Mol Ther 2007, 9(3):248-257.

36. Svoboda P: Cloning and sequencing an inverted repeat. Cold Spring Harb Protoc. 2009, 2009(1):pdb ip64.

37. Parsons BD, Schindler A, Evans DH, Foley E: A direct phenotypic comparison of siRNA pools and multiple individual duplexes in a functional assay. PLoS One 2009, 4(12):e8471.

38. Wang L, Ogburn CE, Ware CB, Ladiges WC, Youssoufian H, Martin GM, Oshima J: Cellular Werner phenotypes in mice expressing a putative dominant-negative human WRN gene. Genetics 2000, 154(1):357-362.

39. Chatot $\mathrm{CL}$, Ziomek CA, Bavister BD, Lewis JL, Torres I: An improved culture medium supports development of random-bred 1-cell mouse embryos in vitro. J Reprod Fertil 1989, 86(2):679-688.

40. Nassenstein C, Kwong K, Taylor-Clark T, Kollarik M, Macglashan DM, Braun A Undem BJ: Expression and function of the ion channel TRPA1 in vagal afferent nerves innervating mouse lungs. J Physiol 2008, 586(6):1595-1604.

41. Tichopad A, Dilger M, Schwarz G, Pfaffl MW: Standardized determination of real-time PCR efficiency from a single reaction set-up. Nucleic Acids Res 2003, 31(20):e122.

42. Pfaffl MW, Horgan GW, Dempfle L: Relative expression software tool (REST) for group-wise comparison and statistical analysis of relative expression results in real-time PCR. Nucleic Acids Res 2002, 30(9):e36.

\section{doi:10.1186/1477-5751-9-8}

Cite this article as: Sarnova et al:: Shortcomings of short hairpin RNAbased transgenic RNA interference in mouse oocytes. Journal of Negative Results in BioMedicine 2010 9:8.

\section{Submit your next manuscript to BioMed Central and take full advantage of:}

- Convenient online submission

- Thorough peer review

- No space constraints or color figure charges

- Immediate publication on acceptance

- Inclusion in PubMed, CAS, Scopus and Google Scholar

- Research which is freely available for redistribution

Submit your manuscript at www.biomedcentral.com/submit
Biomed Central 